\title{
Diversifizierung der Programme der Stadt- und Staatstheater als Reaktion auf die veränderte Stadtgesellschaft?
}

\section{Hilko Eilts}

\section{Zusammenfassung}

Dass die Stadt- und Staatstheater nicht in der Lage seien, sich auf die neuen Realitäten einer pluralisierten Stadtgesellschaft einzustellen und ihre Rolle neu zu definieren, sie stattdessen in Traditionen verharrten und, ,von allem zu viel und immer das gleiche" spielten, ist ein im Rahmen der Stadtheaterdebatte häufig geäußerter Vorwurf. Ein sehr viel komplexeres und widersprüchlicheres Bild ergibt sich unter anderem aus der Analyse der Werkstatistiken des Deutschen Bühnenvereines wie auch der Theaterstatistiken. Sie legen nahe, dass sich seit Beginn der 1990er Jahre bedeutende Veränderungen in den Repertoires und Spielplänen der Stadt- und Staatstheater vollzogen haben, die sich als Versuch der Theater interpretieren lassen, sich neu in der Stadtgesellschaft der Gegenwart zu verorten. Der Aufsatz arbeitet die Bemühungen der Theater um eine größere Repertoirevielfalt und Neuverortung heraus, um anschlieBend die Frage zu diskutieren, welche Schlüsse sich hieraus sowohl für die Kulturpolitik wie auch die Theater ziehen lassen.

\section{Schlüsselwörter}

Theaterprogramme • Diversifizierung • Stadtgesellschaft • Werkstatistik • Repertoire

H. Eilts $(\bowtie)$

Hochschule Hannover, Hannover, Deutschland

E-Mail: hilko.eilts@hs-hannover.de 


\section{$1 \quad$ Einleitung}

Dass die deutschen Stadttheater als historisch gewachsene, hochsubventionierte Einrichtungen nicht in der Lage seien, angemessen auf die sich immer schneller verändernden gesellschaftlichen und künstlerischen Realitäten des 21. Jahrhunderts zu reagieren, sie sich schwer tun, ihren Platz in der von wachsender Diversität geprägten heutigen Stadtgesellschaft zu finden, ist ein in der Stadttheaterdebatte häufig geäußerter Vorwurf. ,Von allem zu viel und nahezu überall das Gleiche" (Haselbach et al. 2012, S. 11 f.), stellen Haselbach, Klein, Knüsel und Opitz in ihrer Kulturinfarkts-Polemik mit Blick auf Deutschlands Kulturlandschaft im Allgemeinen und die deutsche Stadttheaterlandschaft im Besonderen fest. Statt auf stete Veränderung sei alles darauf ausgerichtet, dass es so bleibe, ,wie man es, ausgehend vom Geheimrat Goethe, überzogen mit bürgerlicher Gesellschaftspädagogik, instrumentalisiert von den Sinnspendern der siebziger Jahre, eingerichtet habe" (ebd.: S. 11 f.).

Aber auch von Theaterkünstler*innen selber ist in den vergangenen Jahren eine in Teilen ähnliche Kritik am Stadttheatersystem geübt worden, wie etwa von der Dramatikerin Sybille Berg während der Tagung der Intendant*innengruppe des Deutschen Bühnenvereins 2017. Berg konstatiert, die Repertoires der Stadtund Staatstheater beständen im Wesentlichen aus wohlbekannten Klassikern, für die sich die Theaterleitungen bei ihren Spielzeitplanungen immer aufs Neue entscheiden würden - „was zum Mitmurmeln“ (Berg 2020).

„Ein Jahr nach der Entscheidung, etwas Bekanntes zeitgemäß neu zu inszenieren, was ja immer auch eine krasse Provokation bedeutet, reisen die ungefähr 15 aktuellen Starregisseure von einem Theater zum anderen und schrubben nacheinander politische Inszenierungen der immer gleichen Autoren weg, die dem jeweils fast aktuellen außenoder innenpolitischen Thema angepasst werden" (ebd.).

In seinem Aufsatz „Der Kompromiss ist ein schlechter Regisseur“ beschreibt der Komponist, Musiker und Regisseur Heiner Goebbels die Stadttheater als Museen, wobei es aus seiner Sicht deren institutionelle Gravitationskräfte und nach dem Fließbandprinzip organisierte Produktionsprozesse sind, die die Entwicklung künstlerischer Innovation verhinderten. Vorherrschend sei ein ,„̈sthetischer Konventionalismus und Konformismus“ (Goebbels 2008, S. 19 ff.). Auch der Regisseur Matthias von Hartz beschreibt das deutsche Stadttheater als Fabrik, ,die sehr professionell und spezialisiert ein Produkt herstellt“" (von Hartz 2011, S. 31). Dadurch, dass der gesamte Produktionsprozess innerhalb des Systems Stadttheater geschehe, seien die institutionellen Zwänge so groß, dass freie und innovative 
künstlerische Prozesse nicht möglich seien. Es habe sich in Deutschland mit dem die Theaterlandschaft dominierenden Stadtheater eine Monokultur etabliert, die Innovation und Vielfalt nur in geringem Maße ermögliche:

„Wir investieren etwa 90 Prozent der öffentlichen Gelder für die darstellenden Künste in das Stadt- und Staatstheatersystem. Die Erfahrungen der letzten Jahre legen nahe, dass die Zukunft des Theaters, zumindest was Innovation angeht, nicht zu 90 Prozent, sondern eher zu 10 Prozent aus dem Stadttheater kommt“ (ebd., S. 30).

Diese von von Hartz unterstellte Monokultur wird im Stadtheaterdiskurs nicht nur unter künstlerischem Gesichtspunkt problematisiert, sondern auch unter dem Aspekt der sozialen Funktion des Stadtheaters in der heutigen Stadtgesellschaft, wie dies der Theaterwissenschaftler Günther Heeg in seinem Aufsatz „Die Auflösung des Stadttheaters" tut:

„Die heutige Stadtgesellschaft ist fragmentiert, pluralistisch und heterogen. Das Modell der Marktplatz-Öffentlichkeit, in der das Theater seinen Platz hatte, gleich neben dem Rathaus, der Kirche, dem Museum usw. hat sich dezentralisiert und partikularisiert in viele konkurrierende Öffentlichkeiten, die um Anerkennung ringen. Die immer schon scheinhafte Homogenität der bürgerlichen Nationalkultur sieht sich durch die offensichtliche und greifbare kulturelle Hybridisierung, durch Sub- und Parallelkulturen ihres Scheins beraubt“" (Heeg 2013, S. 234).

Von allen genannten Autor*innen wird die Legitimation des Stadtheaters infrage gestellt und dessen grundlegende Umstrukturierung gefordert. Heiner Goebbels etwa schlägt vor, einige der dem Repertoire- und Ensemblebetrieb verpflichteten Häuser umzuwandeln in künstlerische Labore, die, so Goebbels, weitgehend befreit wären von institutionellen Schwerkräften (Goebbels 2008, S. 21). Heeg fordert ein „Stadtheater als transkulturelles Theater“, das den Alleinvertretungsanspruch, das erste Theater einer Stadt zu sein, verliere und sich auflöse in einer neu entstehenden diversen Stadttheaterlandschaft, in der es dann lediglich ,Teil unter Teilen, ein Haus unter anderen Häusern“" sei (Heeg 2013, S. 240 f.).

Die Frage, der im Folgenden nachgegangen werden soll, ist, ob sich die These von einer theatralen Monokultur, vom musealen Charakter des deutschen Stadttheatersystems, seiner mangelnden Relevanz für eine sich pluralisierende Stadtgesellschaft sowie seiner weitgehenden Resistenz gegen jedwede Veränderung anhand empirischer Daten verifizieren lässt.

Als wichtiger Anhaltspunkt für die Beantwortung dieser Frage können die Entwicklungen im Bereich der Theaterrepertoires während der letzten drei Jahrzehnte gelten. Analysiert werden deshalb einerseits Daten der Werkstatistik ,Wer 
spielte was?“ des Deutschen Bühnenvereins für die Spielzeit 2017/2018, die mit denen für die Spielzeit 1991/1992 verglichen werden - der Zeit also, in der die in Hausschließungen und Theaterfusionen kulminierende Krise des Deutschen Stadttheatersystems zu eskalieren begann und auch den Theaterleitungen klar wurde, dass grundlegende Veränderungen unumgänglich sein würden.

Kombiniert und in eine Relation gesetzt werden diese Daten mit einigen Kennzahlen der Theaterstatistik des Deutschen Bühnenvereins für dieselben beiden Spielzeiten. ${ }^{1}$ Da sich besonders in den Langzeitverläufen der Daten die ,großen Bögen“"von Veränderungsprozessen zeigen, wird selektiv auch auf die Theaterstatistikdaten für die Spielzeit 1967/1968 zurückgegriffen. Einwenden ließe sich an dieser Stelle nicht ganz zu Unrecht, dass die Datengrundlage der Werkstatistik eine etwas andere ist als die der Theaterstatistik. Denn sie erfasst neben den Stadt-, Staatstheatern und Landesbühnen auch die aus der Sicht des Bühnenvereins wesentlichen privaten Bühnen sowie einige produzierende Festivals. Da der Löwenanteil der Daten jedoch von den Stadttheatern, Staatstheatern und Landesbühnen kommt, sind die Daten trotz eines gewissen Zerreffektes m. E. sehr aussagekräftig in Bezug auf Entwicklungen im Stadttheatersystem während der letzten dreißig Jahre.

Acht signifikante Tendenzen, die sich bei der vergleichenden Analyse der Werk- und Theaterstatistiken für die Spielzeiten 1991/1992 und 2017/2018 herauskristallisiert haben, sollen im Folgenden schlaglichtartig beschrieben werden. In einem weiteren Schritt werden die werk- und inszenierungsbezogenen Daten dann in eine Relation gestellt zu weiteren Theaterstatistik-Daten, etwa denen zu Theaterbesuchen, Veranstaltungen oder Spielstätten, die ein umfassenderes Bild nicht allein der Veränderung der Theaterprogramme, sondern auch der Gesamtsituation der Theater vermitteln sollen.

\footnotetext{
${ }^{1} \mathrm{Zu}$ den Eigenheiten des deutschen Stadttheatersystem gehört nicht nur der Repertoire- und Ensemblebetrieb, sondern auch eine bis in die 1850er Jahre zurückreichende Tradition der so umfassenden wie ambitionierten statistischen Erfassung des Theaterwesens. Längsschnittanalysen dieser Daten gewähren tiefe Einblicke in den Wandel des Stadttheatersystems insgesamt wie auch in die Entwicklung jedes einzelnen Theaters. Umso mehr verblüfft, dass diese Daten sowohl seitens der Theater als auch seitens der Kulturpolitik in Gänze ungenutzt bleiben und der Deutsche Bühnenverein die wissenschaftliche Erschließung insbesondere der Theaterstatistiken mehr behindert, als dass er sie fördert. Eine vergleichende Analyse der Theaterstatistiken ist letztmalig 1985 unternommen worden.
} 


\section{Die Veränderung der Programme von Stadt-/Staatstheatern und Landesbühnen seit den 1990er Jahren}

\section{1 (Schein-)Kontinuitäten}

Wenn alljährlich die Werkstatistik „Wer spielte was?“ des Deutschen Bühnenvereins veröffentlicht und in einer Vorab-Pressemeldung die Liste der am häufigsten gespielten Werke mit den höchsten Besuchszahlen publiziert wird, scheinen die Daten die Kritiker*innen des Stadttheatersystems zu bestätigen. Auf den Listen der 25 Werke mit den höchsten Inszenierungs- und Besuchszahlen finden sich ein und dieselben Titel seit Jahrzehnten beharrlich wieder: Mozarts Zauberflöte, Goethes Faust, Shakespeares Sommernachtstraum, Bizets Carmen, Humperdincks Hänsel und Gretel etc. Wandel, Innovation, Vielfalt sucht man vergebens. Selbst der geschäftsführende Direktor des Bühnenvereins stellt in einer Pressemeldung kritisch fest:

„Diese Zahlen zeigen, dass es noch ein weiter Weg ist, bis gesellschaftliche Veränderungen in den Spielplänen der Theater auftauchen: Das gilt sowohl für die Geschlechtergerechtigkeit wie für das Zugehen der Theater auf neue Publikumsschichten, aber auch für den Umgang mit enttäuschten Abonnent*innen. Eine Weiterentwicklung der Theaterspielpläne wird unumgänglich sein“ (Deutscher Bühnenverein 2019c).

Ein wesentlich komplexeres, von vielfachen Ambiguitäten und Ambivalenzen geprägtes Bild als vom Bühnenverein selber suggeriert, ergibt sich dagegen, wenn man die umfangreichen aktuellen Daten der Werkstatistik im Detail analysiert, die Listen der Autor*innen und Werke durchgeht und sie vergleicht mit denen einer Werkstatistik von Anfang der 1990er Jahre.

\subsection{Mehr Mehr Mehr! Die wachsenden Repertoires}

Begonnen werden soll mit zwei Kennzahlen aus der Theaterstatistik: In der Rubrik „Veranstaltungen“ finden sich dort neben den nach Sparten getrennt aufgeführten Veranstaltungen die „Sonstigen Veranstaltungen“ und Veranstaltungen des „theaternahen Rahmenprogramms“. Es finden sich aber auch Angaben zu den Inszenierungen und Neuinszenierungen einer Spielzeit, die das Gesamtrepertoire der erfassten Theater ausmachen. 


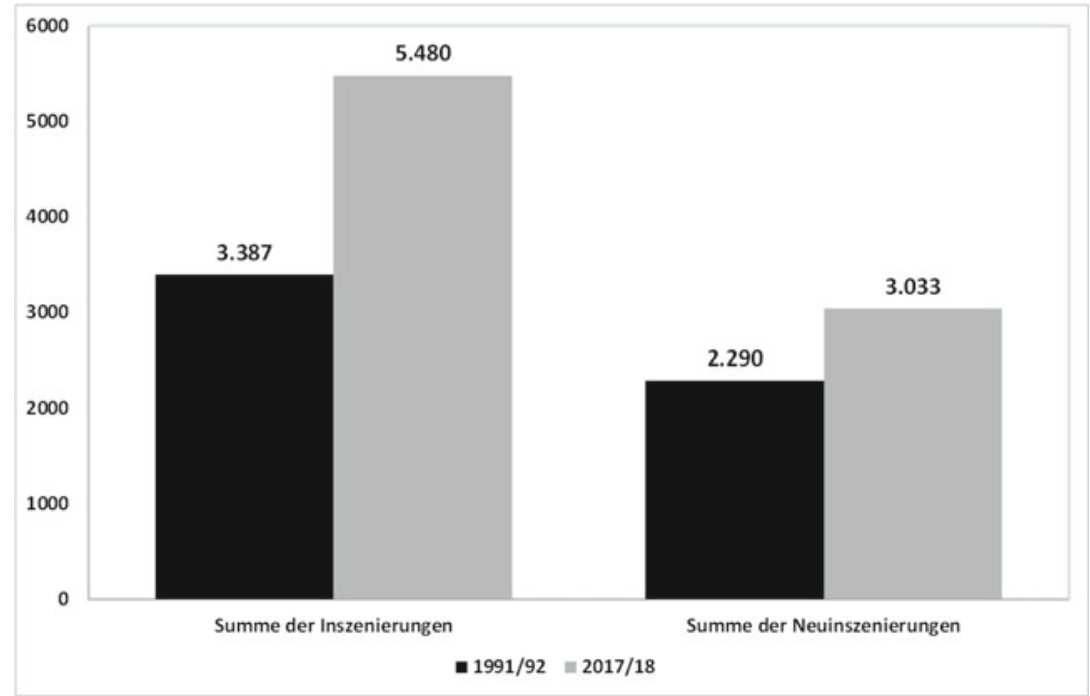

Abb. 1 Inszenierungen und Neuinszenierungen an den deutschen Stadt-/Staatstheatern und Landesbühnen für die Spielzeiten 1991/1992 und 2017/2018 im Vergleich. (Quelle: Theaterstatistik des DBV, Jahrgänge 1991/1992 und 2017/2018. (C Hilko Eilts, 2020)

Aus ihnen lässt sich errechnen, dass in der Spielzeit 1991/1992 ein Theater im Schnitt 22 Inszenierungen im Programm hatte, von denen 15 Neuinszenierungen waren (vgl. Abb. 1). In der Spielzeit 2017/2018 waren es dagegen 39 Inszenierungen pro Theater (das ist ein Plus von $77 \%$ ) und 21 Neuinszenierungen (das ist ein Plus von $40 \%$ ). Das Inszenierungsangebot, das die Theater ihrem Publikum machten, ist also signifikant gestiegen. 2017/2018 wurden bedeutend mehr Produktionen von den Theatern realisiert als 1991/1992 und damit ein entsprechender Mehraufwand betrieben, da für jede einzelne Produktion Regieteams engagiert, Bühnenbilder gebaut, Kostüme angefertigt, Probenzeiträume und Bühnenzeiten zur Verfügung gestellt werden müssen. Das häufig gezeichnete Bild des immer mehr produzierenden, immer mehr Angebote schaffenden und sich dabei verausgabenden Stadttheaters wird also von den Statistiken des Bühnenvereins durchaus gestützt. ${ }^{2}$ Aber wird auch überall das Gleiche produziert?

\footnotetext{
${ }^{2}$ Siehe hierzu etwa Schmidt 2017, S. 91 ff. oder Höhne 2019, S. 15.
} 


\subsection{Die neue Vielfalt der Repertoires}

Auskunft hierüber gibt die Werkstatistik, die im Gegensatz zur Theaterstatistik nicht nur erfasst, wie viel gespielt wird, sondern auch was. Aufgeführt werden autor*innenbezogen sämtliche in einer Spielzeit gespielten Werke, die Anzahl und Orte ihrer Inszenierungen, die Anzahl der Aufführungen sowie der Besuche pro Inszenierung. Umfasste in der Spielzeit 1991/1992 das Gesamtrepertoire der erfassten Theater in Deutschland 2800 Werke, waren es in der Spielzeit 2017/2018 insgesamt 4779, das bedeutet eine Zunahme der aufgeführten Werke um $79 \%$. Und anders als die Best-off-Liste nahelegt, offenbart die Detailanalyse signifikante Veränderungen in Bezug auf die Gestalt des Gesamtrepertoires.

\subsection{Weniger Klassiker(pflege)}

Zum einen hat die Bedeutung und Bindekraft der Klassiker und der zentralen Positionen in den Spielplänen der Theater deutlich nachgelassen. Wurden 1991/1992 die zwanzig meistgespielten Werke noch 10.450 Mal aufgeführt und kamen auf 7,2 Mio. Besuche, so waren es in der Spielzeit 2017/2018 nur 5728 Aufführungen (minus $46 \%$ ), die 4,2 Mio. Besuche auf sich vereinten (minus $41 \%$ ). Die Werke eines Shakespeares, Molières, Schillers, Brechts, eines Mozarts, Wagners, Bizets oder Verdis werden heute deutlich weniger oft aufgeführt, inszeniert und besucht als vor 30 Jahren. Die Funktion der Stadtheater als Einrichtungen zur Klassikerpflege und Kulturgutvermittlung hat nachweisbar an Relevanz abgenommen.

Am meisten zu spüren bekommt diese Entwicklung die Sparte, die der Klassikerpflege und Kulturgutvermittlung besonders stark verbunden ist: das Musiktheater, dessen Repertoire sich im Wesentlichen aus etwa zwei Dutzend Werken zusammensetzt, die zum großen Teil aus dem 18. und 19. Jahrhundert stammen. ${ }^{3}$ Darüber hinaus haben Oper und Operette, gemessen an Inszenierungs-, Aufführungs- und Besuchszahlen, mit einem dramatischen Relevanzverlust zu

\footnotetext{
${ }^{3}$ Laut Werkstatistik für die Spielzeit 2017/2018 belief sich der Anteil der nichtzeitgenössischen Opernwerke an der Gesamtzahl der Opernaufführungen in Deutschland auf knapp $84 \%$, der Anteil der Uraufführungen lag lediglich bei $4 \%$. Damit ist das Musiktheater im Vergleich zu Schauspiel, Tanz und Kinder- und Jugendtheater die künstlerisch am wenigsten innovative Sparte.
} 
kämpfen. ${ }^{4}$ Auch hinsichtlich der Publikumsstruktur steht das Musiktheater vor schwerwiegenden Problemen, wie zahlreiche Studien belegen. ${ }^{5}$

\subsection{Mehr Zeitgenössisches}

Entsprechend stark zugenommen hat hingegen die Relevanz des Zeitgenössischen. Ganze 196 Uraufführungen zählte die Werkstatistik 1991/1992, zwanzig Jahre später waren es bereits 587, in der aktuellen Ausgabe für die Spielzeit 2017/2018 findet sich die Zahl von 1141 - das ist eine knappe Versechsfachung im Bereich der Uraufführungen. ${ }^{6}$

\footnotetext{
${ }^{4}$ Eindringlich belegen vergleichende Analysen der Theaterstatistiken den Relevanzverlust von Oper und Operette sowohl gesamtgesellschaftlich als auch bezogen auf die anderen Sparten der Theater. Werden in der Theaterstatistik für die Spielzeit 1991/1992 6,1 Mio. Besuche für Oper und Operette gezählt, sind es in der Theaterstatistik für die Spielzeit 2017/2018 mit 4,2 Mio. 1,9 Mio. Besuche weniger (minus $31 \%$ ). Lag der Anteil der Opern- und Operettenbesuche an der Gesamtbesuchszahl der Theater (Besuche der eigenen und fremden Veranstaltungen am Standort) in der Spielzeit 1991/92 bei $31 \%$, so ist er in der Spielzeit 2017/2018 auf $23 \%$ gesunken. Geht man bei der vergleichenden Analyse 50 Jahre zurück (Spielzeit 1967/1968), kommt man bei den Opern- und Operettenbesuchen in der alten BRD auf erstaunliche 8,6 Mio, die $44 \%$ der Gesamtbesuche ausmachten. In der alten Bundesrepublik des Jahres 1968 mit ihren knapp 60 Mio. Einwohner*innen sind Oper und Operette folglich mehr als doppelt so häufig besucht worden als in der neuen Bundesrepublik mit ihren 82,8 Mio. Einwohner*innen.

${ }^{5}$ Siehe u. a. hierzu auch Reuband 2011, S. 397 f., Rössel und Hölscher 2018, S. 241 oder Höflich 2018, S. 281. „Die Deutsche Bühne“ widmete 2016 dem Thema Oper unter dem Titel „Oper öffnen“ einen Themenschwerpunkt, der die hier angedeuteten Probleme ausführlich thematisierte und in dem Detlef Brandenburg konstatierte: „Die Oper hat derzeit ein Publikum, das älter und homogener ist als das des Schauspiels oder des Tanzes. Sie ist die Theatersparte, die in ihrer Publikumsstruktur am schwächsten auf den demografischen Wandel und die enorme Beschleunigung von Migration, sozialer Mobilität und digitaler Kommunikation reagiert. Das kann auf lange Sicht durchaus zu einem Relevanz- und am Ende zu einem Akzeptanzverlust führen.“ (Brandenburg 2016b, S. 50).

${ }^{6}$ Zwar können auch sogenannte „Ausgrabungen“, d. h. ältere Werke, die noch nie aufgeführt worden sind, als Uraufführungen zählen. Dies ist aber die Ausnahme. Sehr überwiegend handelt es sich bei den „Uraufführungen“ in der Tat um zeitgenössische Werke.
} 


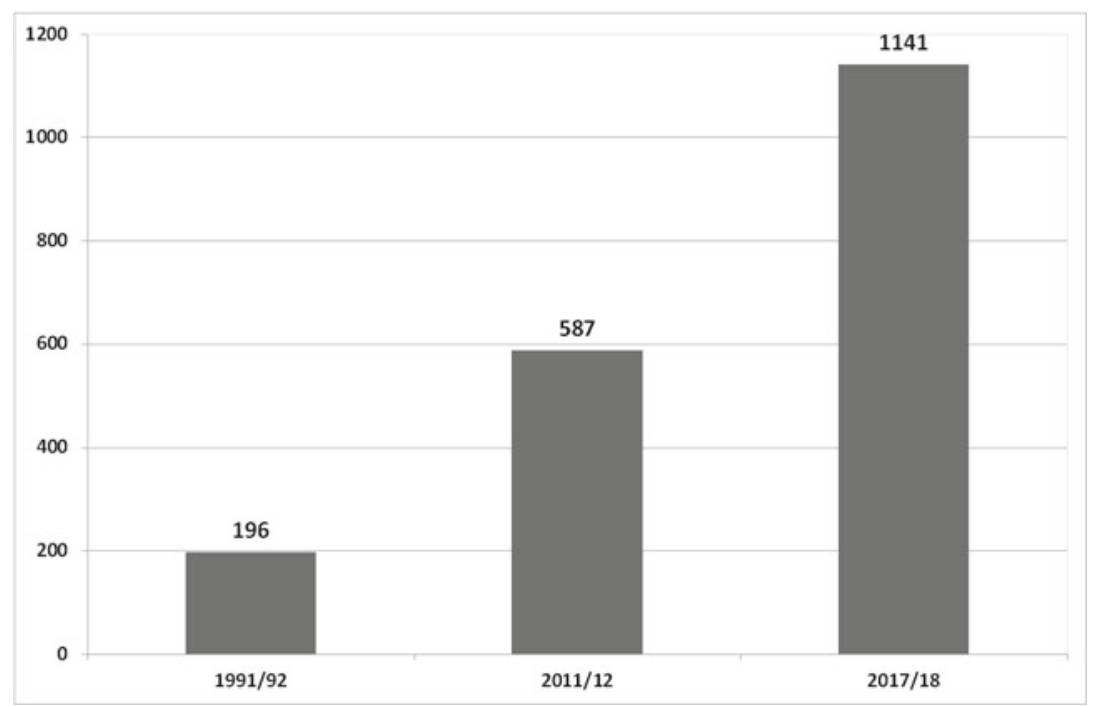

Abb. 2 Anzahl der Uraufführungen an den deutschen Stadt-/Staatstheatern, Landesbühnen sowie erfassten Privattheatern in den Spielzeiten 1991/1992, 2011/2012 und 2017/2018 im Vergleich. (Quelle: Werkstatistik des DBV, Jahrgänge 1991/1992, 2011/2012 und 2017/2018. (C) Hilko Eilts, 2020)

Vor diesem Hintergrund ist es nicht übertrieben, von einem sukzessiven Haltungswechsel bei den Theatern in Relation zu den frühen 1990er Jahren zu sprechen, einer deutlich höheren Bereitschaft, sich im Hier und Heute zu verorten, wofür noch eine andere Zahl spricht. Glaubt man den Angaben der Werkstatistik, waren in der Spielzeit 2017/2018 gut $80 \%$ aller inszenierten Werke zeitgenössische, also solche, die ihr Uraufführungsdatum nach 1945 hatten (vgl. Abb. 2).

\subsection{Neue Formen, Formate und Genres oder: Postdramatik als Breitenbewegung}

Statistisch dokumentiert ist auch die veränderte Haltung der Theater zum Dramentext, ja zum Text und zur Textvorlage grundsätzlich, die zu einer neuen Vielfalt 
der Formen und Formate geführt hat. In einem der Werkstatistik 2014/2015 vorangestellten Essay schreibt Detlef Brandenburg, Chefredakteur der „Deutschen Bühne" dazu:

\begin{abstract}
„Theater gehen immer mehr dazu über, Produktionen zu spielen, die zwar oft noch auf einer eindeutig identifizierbaren Stoffgrundlage beruhen. Aber selbst dann, wenn diese Vorlage tatsächlich ein dramatischer Text ist, wird sie keineswegs mehr verbindlich für die ästhetisch formale Struktur der Aufführung, sondern sie dient dieser nur mehr als Material. Daneben gibt es Produktionen, die ihre Text- und Handlungsversatzstücke aus unterschiedlichen Vorlagen collagieren. Und schließlich kommt auch die Kategorie der nach traditionellem Verständnis völlig, werkfreien ' Produktionen immer häufiger vor, die ihr Spiel- und Sprachmaterial erst während des Probenprozesses erarbeiten oder aber zuvor durch individuell auf die Produktion bezogene Rechercheprozesse generieren." (Brandenburg 2016a, S. 8)
\end{abstract}

Brandenburg meint eine Verschiebung des Werkbegriffs in der Breite beobachten zu können. Die sehr unterschiedlichen Werk-Manifestationen in diversen Theaterformaten führten dazu, dass, anders als noch vor wenigen Jahrzehnten, eine auf der Höhe ihrer Zeit agierende Theaterstatistik gar nicht mehr a priori definieren könne, was ein Werk zu sein habe (Ebd.: S. 8).

Folgerichtig hat der Bühnenverein vor fünf Jahren die Werkstatistik in Teilen umgearbeitet und unter anderem die neuen Kategorie der „Bearbeitung“ sowie „Projekte/Mehrspartenprojekte/Performance“ eingeführt. Als „Bearbeitungen“ gelten in der Werkstatistik 2017/2018 ein Drittel aller Inszenierungen im Schauspiel. Im Kinder- und Jugendtheater sind es fast drei Viertel. Ins Auge fallen besonders die zahlreichen Romandramatisierungen aber auch Bearbeitungen von Filmstoffen. Unter das neu eingeführte Genre „Projekt/Mehrspartenprojekt/Performance “ fallen immerhin $12 \%$ aller Inszenierungen der erfassten deutschen Theater. $\mathrm{Zu}$ dieser Kategorie werden auch viele der Bürgerbühnenprojekte gezählt, die eine weitere Tendenz markieren.

\title{
2.7 Mehr Bezug zur Stadt
}

$\mathrm{Zu}$ konstatieren ist ein Trend zur unmittelbaren Auseinandersetzung mit den Realitäten der heutigen Stadtgesellschaft, sei es in Form von stadtbezogenen Recherche- und Dokumentartheaterprojekten, Bürgerbühnen, Mehrgenerationenensembles oder zahlreichen interaktiven Formaten. Unter der Kategorie „Projekt/Mehrspartenprojekt/Performance“ finden sich etwa Titel wie „New Hamburg“ von Björn Bicker (Schauspielhaus Hamburg), „Stadt Utopia“ am Theater Lübeck 
von der Kompanie Kopfstadt und Charlotte Baumgart und Jeffrey Dörings „Rockstock Revolution Road“ am Volkstheater Rostock, das „Mannheim Experiment“ am Nationaltheater Mannheim von der Bürgerbühne Mannheim, ein Projekt mit Geflüchteten mit dem Titel „Der Hauptmann von O, eine Kopenickiade“ am Theater Osnabrück, um nur einige zu nennen.

All diesen Projekten ist gemeinsam, dass es nicht mehr der tradierte, weitgehend fixe und vorgängige Dramentext ist, der von der Regie in Szene gesetzt und vom Ensemble des Theaters allabendlich aufgeführt wird. Vielmehr werden viele dieser Projekte aus dem spezifischen Kontext der Stadtgesellschaft heraus und vor dem Hintergrund aktueller gesellschaftlicher und lokaler Fragen nicht selten mit Bürger*innen oder anderen Akteur*innen einer Stadt auf unterschiedlichste Weise entwickelt und zur Aufführung gebracht. Die vermehrten Bemühungen um das Publikum vor Ort als die zentrale Anspruchsgruppe der Theater kommen darüber hinaus in dem außerordentlichen Zuwachs der ,vermittelnden“ Formate zum Ausdruck, die sich in den Theaterstatistiken unter der Rubrik ,theaternahes Rahmenprogramm“ finden. ${ }^{7}$

\subsection{Die lange nicht wahrgenommene, unterschätzte Sparte: Das Kinder und Jugendtheater}

Aber noch ein weiteres eigenständiges Genre wurde in der neu organisierten Werkstatistik etabliert, das in Zusammenhang mit der veränderten Haltung vieler Stadt- und Staatstheater gegenüber der Stadtgesellschaft von großer und weitgehend unterschätzter Bedeutung ist: das Kinder- und Jugendtheater. Es hat im Gegensatz zum Musiktheater, aber auch zum Schauspiel, seit Anfang der 1990er Jahren kontinuierlich an Bedeutung gewonnen, sei es auf der Ebene der Veranstaltungszahlen (plus $87 \%$ ), der Besuchszahlen (plus $34 \%$ ) oder der Inszenierungszahlen. Der hohe Anteil von Uraufführungen an den Inszenierungen des Kinder- und Jugendtheaters, der in der Werkstatistik 2017/2018 auf knapp 17 \% beziffert wird, sowie ein Anteil von knapp $48 \%$ Bearbeitungen zeigt, dass diese Sparte des Theaters insbesondere seit den 1990er Jahren ein starkes, eigenständiges und vielfältiges künstlerisches Profil gewonnen hat. ${ }^{8}$ In den letzten drei

\footnotetext{
${ }^{7}$ Siehe den Beitrag von Bianca Michaels in diesem Band.

${ }^{8}$ Israel (2009) stellt hierzu fest: „Das Kinder- und Jugendtheater hat sich als freie Szene, als kommunales oder staatliches Theater und als vierte Sparte etabliert und als Kunst ästhetisch vielgestaltig emanzipiert. Es will durch seine Kunst wirken. Es ist Theater geworden (fast) wie jedes andere (...)“ (Israel 2009, S. 28).
} 
Jahrzehnten ist das Kinder- und Jugendtheater zu einer zentralen Säule im Spielbetrieb der Stadttheater geworden, die nach dem Schauspiel und der Oper die drittmeisten Theaterbesuche generiert. Die Arbeit mit Kindern und Jugendlichen, die ebenso wie der zeitgenössische Tanz- und Performancebereich lange Zeit eine wichtige Nische der „freien Theaterszene“"war, hat für die Stadtheater eine neue Priorität gewonnen, die sich in Jugendclubgründungen, eigenständigen Kinderund Jugendtheatersparten, der Einführung von Kooperationsverträgen mit Schulen etc. niedergeschlagen hat. ${ }^{9}$

\section{Die Veränderungen der Programme im Kontext der Entwicklung von Besuchszahlen, Theaterressourcen und -strukturen}

Anders als von Kritiker*innen des Stadttheatersystems unterstellt, haben sich seit den 1990er Jahren nachweislich bedeutende Veränderungen in den Programmen der Häuser vollzogen:

- Die Stadt-/Staatstheater und Landesbühnen haben ihre Angebote seit den 1990er Jahren bedeutend vergrößert, sie produzieren insgesamt signifikant mehr, wobei das Bild von Sparte zu Sparte durchaus variieren kann.

- Es wurde eine neue Angebotsvielfalt mit neuen Veranstaltungsformen, Genres und Ästhetiken mit deutlich mehr Gegenwartsbezug geschaffen, die in Teilen auf eine größere Interaktion zwischen Stadtgesellschaft und Theater zielen. Diese Veränderungen der Theaterrepertoires lassen sich als Analogbewegungen zu den (stadt-)gesellschaftlichen Diversifizierungsdynamiken lesen. Es lässt sich hier die Hypothese formulieren, dass die Theater versuchen, den Erwartungshaltungen möglichst vieler Anspruchsgruppen gerecht zu werden sowie auf möglichst vielen Feldern des Legitimationsdiskurses Punkte zu sammeln, sei es in Bezug auf die Fähigkeit zu künstlerischer Innovation bei

\footnotetext{
${ }^{9}$ Siehe hierzu den Beitrag von Charlotte Burghardt in diesem Band. Sehr kritisch betrachtet Höhne (2019) diese Entwicklungen, wenn er in Zusammenhang mit dem Anstieg der Besuchszahlen in der Sparte „Kinder- und Jugendtheater“ von einer „,kulturpädagogischen Instrumentalisierung“ und „Funktionalisierung der Hochkultur“ spricht, der ,allerdings ,traditionelle“ kulturpolitische Aufgaben wie Erhalt, Pflege und Weiterentwicklung des ,klassischen“ kulturellen Erbes oder Sammlung ästhetischer Erfahrungen leicht einer Marginalisierung anheimfallen“ könnten (Höhne 2019, 16). Die abwertende Haltung Höhnes, der dem Kinder- und Jugendtheater den Status einer Kunst abspricht, um es einer vermeintlichen „Hochkultur“ gegenüber zu stellen, kann sowohl in Hinsicht auf das Theater als auch die Theaterwissenschaft als exemplarisch gelten.
} 
gleichzeitiger Vermittlung des kulturellen Erbes, die Beteiligung an kultureller Bildung, die Nachwuchsförderung, die Initiierung von politischen Diskursen in der Stadtgesellschaft oder die Beteiligung am „Community Building“ etc. ${ }^{10}$

- Es zeichnen sich Akzent- und Schwerpunktverschiebungen zwischen den Sparten, Genres, Veranstaltungsformen ab. Während in Relation zur Gesamtbesuchszahl insbesondere das Musiktheater aber auch das Schauspiel an Relevanz verloren hat, ist die Bedeutung des Kinder- und Jugendtheaters sowie des Tanzes zwischen 1991 und 2018 gestiegen.

Ganz so veränderungsresistent und indifferent gegenüber den neuen Realitäten einer diversifizierten Stadtgesellschaft wie oft unterstellt, sind die Stadt- und Staatstheater, zumindest in Bezug auf ihre Repertoires, nachweislich nicht, was sich aus Sicht der Theater als gute Nachricht lesen lässt.

Gleichzeitig gehen mit den beschriebenen Entwicklungen aber auch durchaus gravierende Probleme einher, was sich insbesondere dann zeigt, wenn man sie in eine Relation stellt zu weiteren zentralen Kennzahlen aus den Theaterstatistiken der ausgewählten Spielzeiten (vgl. Abb. 3).

- In Hinblick auf die Besuchszahlen zeigt sich, dass all die Bemühungen der Theater in Bezug auf eine Erhöhung der Besuchszahlen nicht erfolgreich waren. Dem beachtlichen Zuwachs an Angeboten und der starken Diversifizierung der Angebotsstruktur steht eine kontinuierliche Abnahme an Theaterbesuchen gegenüber, die in der Spielzeit 2017/2018 in Relation zur Spielzeit 1991/1992 ein neues Allzeittief erreicht hat (minus 7 \%). An dem in Bezug auf die Besuchszahlen gemessenen, bereits Mitte der 1960er Jahre einsetzenden, kontinuierlichen Bedeutungsverlust der Stadt- und Staatstheater konnte auch die deutliche Neuausrichtung der Theaterprogramme bislang nichts ändern. ${ }^{11}$

\footnotetext{
${ }^{10}$ Die Widersprüchlichkeit der Erwartungshaltung der verschiedenen, dem Theater zuzuordnenden Anspruchsgruppen hat von Cossel (2011, S. 46 f.) in ihrer empirischen Studie zur „Entscheidungsfindung im Kulturbetrieb am Beispiel der Spielplangestaltung im Theater“ herausgearbeitet.

${ }^{11}$ Die Abnahme bei den Theaterbesuchen lässt sich als ein langfristiger, sukzessiver Erosionsprozess beschreiben, dessen Dramatik bei einer Längsschnittanalyse der Theaterstatistikdaten und der Berechnung aussagekräftiger Verhältniszahlen deutlich wird. Wurde in der Spielzeit 2017/2018 im Schnitt jedes der erfassten Theater knapp 129.000 Mal besucht, waren es in der Spielzeit 1967/1968 knapp 228.000, also fast 100.000 Male mehr. Gerade bei Theaterneubauund -sanierungsprojekten (Staatstheater Augsburg, Staatsoper Stuttgart, Oper Köln, Städtische Bühnen Frankfurt) findet diese bedeutende Entwicklung in der Regel ebenso wenig
} 


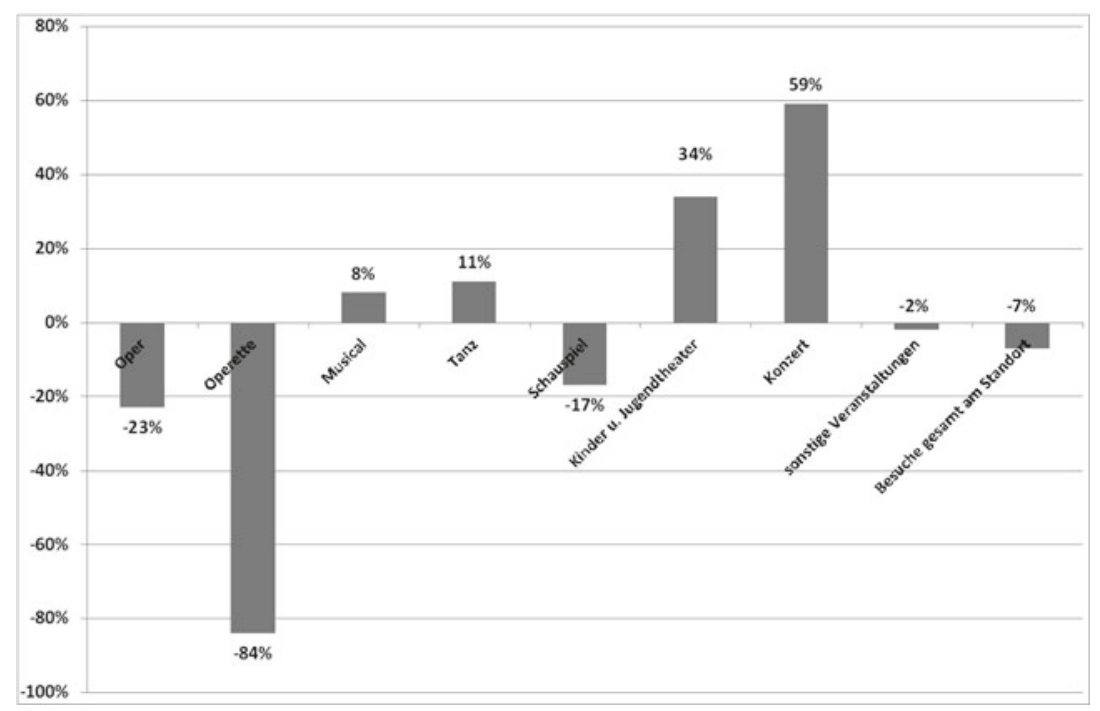

Abb.3 Theaterbesuche Stadt-/Staatstheater und Landesbühnen in den Spielzeiten 1991/1992 und 2017/2018 verteilt auf Sparten und Veranstaltungsarten im Vergleich in Prozent. (Quelle: Theaterstatistik des DBV, Jahrgänge 1991/1992 und 2017/2018. (C Hilko Eilts, 2020)

- Weniger Besuche bedeuten weniger Einnahmen und das führt zu einem weiteren Problem: Dem betriebenen Mehr an Aufwand steht ein Weniger an Ressourcen gegenüber, insbesondere, was das Personal angeht, das seit 1991/1992 um $11 \%$ abgebaut worden ist. Die Prekarisierung der Arbeitsverhältnisse gerade bei den NV-Solo-Beschäftigten sowie die vergleichsweise hohe Unzufriedenheit der Theaterbeschäftigten hinsichtlich der Arbeitsverhältnisse am Theater sind ganz wesentlich auf diese Diskrepanz zurückzuführen. ${ }^{12}$

- Dasselbe gilt für die Produktionsbedingungen und künstlerischen Prozesse. An den beschriebenen Zahlen lässt sich ablesen, dass die von Goebbels und von Hartz problematisierten institutionellen Schwerkräfte in den letzten 30 Jahren deutlich zugenommen haben. So haben sich die durchschnittlichen Proben-,

Berücksichtigung wie die Relevanzverschiebungen zwischen den verschiedenen Theatersparten. Stellt man die Neubau- und Sanierungsprojekte in ein Verhältnis zu den Befunden einer vergleichenden Analyse von Theater- und Werkstatistik, dann wird deutlich, dass die Bauprojekte tendenziell zu groß dimensioniert und zu musiktheaterzentriert ausgerichtet sind. ${ }^{12}$ Siehe den Beitrag von Althoff/Priller/Zimmer in diesem Band. 
Beleuchtungs- und Bühnenzeiten verkürzt, was mit einer immer strikteren Plan- und Zielorientierung der Probenprozesse einhergeht, oder die Ausstattungsetats pro Produktion mit der Folge reduziert, dass Kostüme vielfach nicht mehr angefertigt, sondern aus dem Fundus eines Hauses organisiert werden.

- Weniger Theaterbesuche in Kombination mit mehr Inszenierungen und Veranstaltungen haben zudem dazu geführt, dass im Schnitt pro Inszenierung und Veranstaltung deutlich weniger Menschen sitzen (vgl. Abb. 4). 1991/92 saßen im Schnitt in jeder Theaterveranstaltung 346 Besucher*innen, in der Spielzeit 2017/18 waren es nur noch 280. Verfolgt man die Entwicklung dieser Verhältniszahlen anhand der Theaterstatistiken bis in die Spielzeit 1967/68 zurück, kommt man zu noch drastischeren Unterschieden. Damals waren es 620 Theaterbesucher*innen pro Veranstaltung. Sowohl unter betriebswirtschaftlichen als auch unter Nachhaltigkeitsgesichtspunkten sind diese Entwicklungen als problematisch zu bewerten.

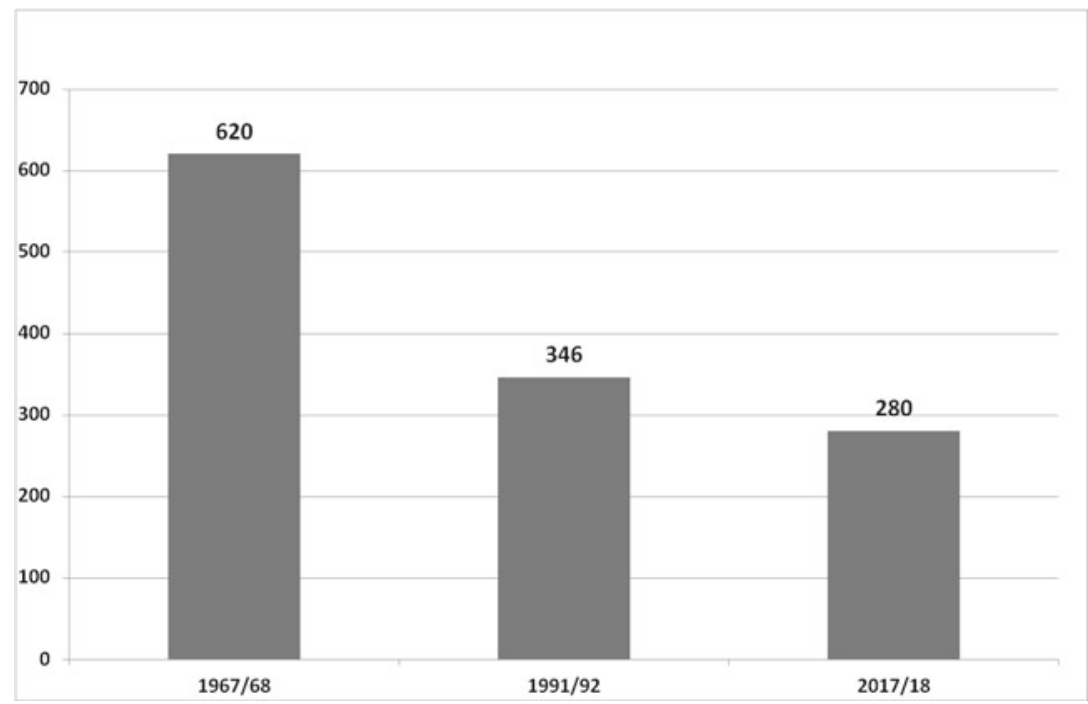

Abb. 4 Besuche pro Veranstaltung der deutschen Stadt-/Staatstheater und Landesbühne für die SZ 1967/1968, 1991/1992 und 2017/2018. (Quelle: Theaterstatistik des DBV, Jahrgänge 1967/1968, 1991/1992 und 2017/2018. @ Hilko Eilts, 2020) 
- Die Bespielung der Theater gestaltet sich bei abnehmender Besuchszahl wesentlich kleinteiliger und das hat weitreichende Konsequenzen. So hat sich die Halbwertszeit von Inszenierungen stark verkürzt (von im Schnitt 17 Vorstellungen zu 11 Vorstellungen). Und noch gravierender: Die Großen Häuser werden zum Problem. In allen im Rahmen meiner Forschungen untersuchten Theatern ${ }^{13}$ hat eine starke Verlagerung der Veranstaltungen und der Besucher*innenströme weg von den Großen Häusern hin zu den mittleren und kleineren Spielstätten stattgefunden, wie hier am Beispiel Bremen deutlich wird (vgl. Abb. 5). ${ }^{14}$

- Die beschriebenen quantitativen wie auch die qualitativen Veränderungen der Programme gehen also einher mit insgesamt stark veränderten Anforderungen an die historisch gewachsenen, auf eine zentrale, großteilige Bespielung ausgerichteten Stadt- und Staatstheaterapparate. Dynamisierung, Diversifizierung und die immer weiter zunehmende Kleinteiligkeit im Bereich der Programme sowie die Dezentralisierung des gesamten Spielbetriebes stellen die technischen Abteilungen ebenso vor neue Herausforderungen wie die Intendanzen, kaufmännischen Direktionen, die Presse- und Öffentlichkeitsabteilungen oder die künstlerisch Beschäftigten der Häuser.

\section{$4 \quad$ Fazit}

Festhalten lässt sich als Fazit, dass die empirischen Daten hinsichtlich der Programmentwicklung bei den Stadt- und Staatstheatern so komplexe wie zutiefst widersprüchliche, von Ambiguitäten, Ambivalenzen und Paradoxien geprägte Dynamiken sichtbar werden lassen. Das Bemühen der Theater, mit der Veränderung ihrer Programme und Repertoires den neuen Realitäten der heutigen Stadtgesellschaft gerecht zu werden, neue Publikumsschichten zu erreichen und künstlerische Innovation vorantreiben $\mathrm{zu}$ wollen, mit der man wiederum der Erwartungshaltung der Fachöffentlichkeit gerecht werden will, steht neben dem

\footnotetext{
${ }^{13}$ In meiner Dissertation mit dem Arbeitstitel ,Zwischen Tradition und Transformation. Das Deutsche Stadttheatersystem seit der Wende" werden neben dem Theater Bremen das Theater Augsburg, das Volkstheater Rostock, das Deutsche Nationaltheater Weimar sowie das Thalia Theater Hamburg untersucht.

${ }^{14}$ Lag in der Spielzeit 1991/1992 die durchschnittliche Zahl der Spielstätten pro Theater noch bei 3, so kamen in der Spielzeit 2017/2018 auf ein Theater im Schnitt 5,7 Spielstätten, 50 Jahre zuvor, in der Spielzeit 1967/1968 waren es noch 2,1.
} 


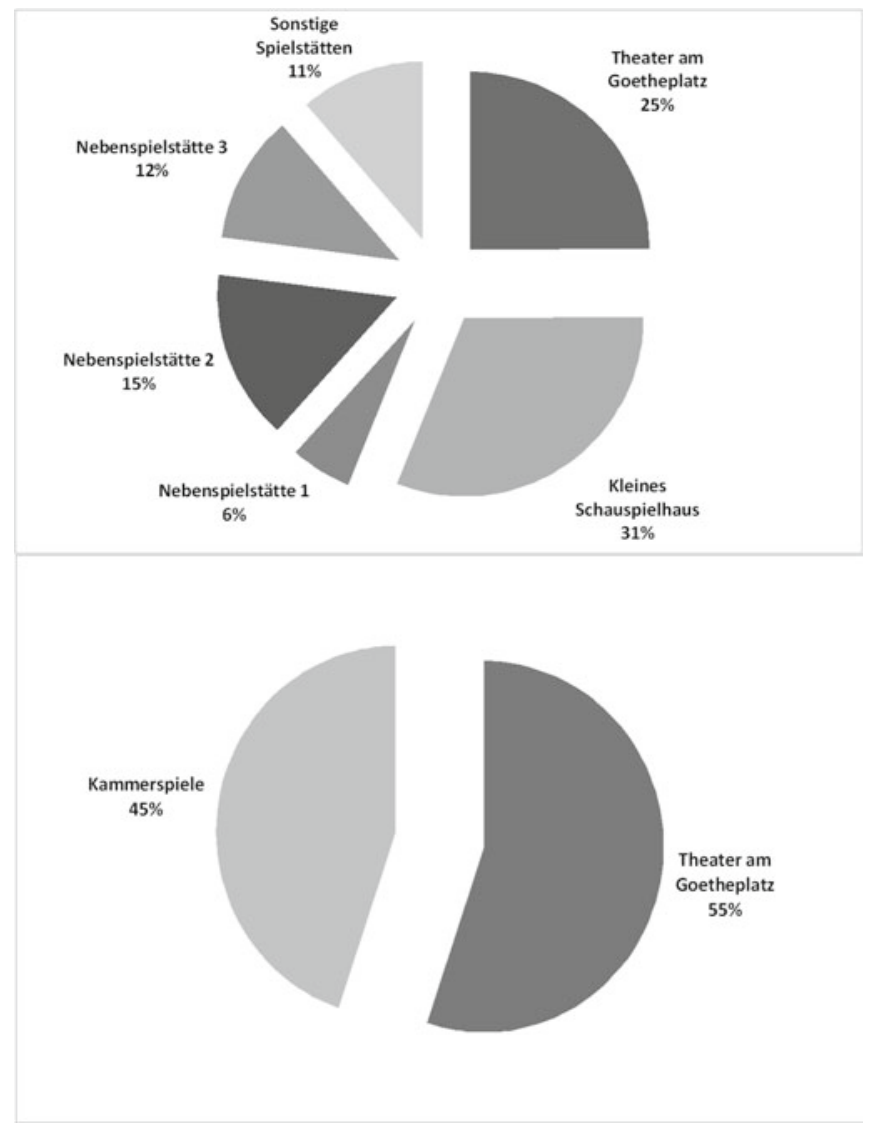

Abb. 5 Theater Bremen, Veranstaltungen am Ort und ihre Verteilung auf die Spielstätten in Prozent in den Spielzeiten 1967/1968 und 2017/2018. (Quelle: Theaterstatistik des DBV, Jahrgang 1967/1968 und 2017/2018. (C) Hilko Eilts, 2020)

gleichzeitigen Bemühen um Kulturpflege und dem Erhalt des Abonnementpublikums. ${ }^{15}$ Diese Tendenzen wiederum stehen im Zeichen eines sich ungebrochen

\footnotetext{
${ }^{15}$ Wie stark die hochgradig widersprüchlichen Anforderungen an die Spielplangestaltungen Intendanzen und Dramaturgien beschäftigen, kommt in zahlreichen Interviews zum Ausdruck, die von von Cossel im Rahmen ihrer empirischen Studie zur Spielplangestaltung geführt wurden (von Cossel 2011, S. 197 ff. sowie 228 f.).
} 
fortsetzenden Publikumsschwundes, reduzierter Ressourcen, dem Zwang zu mehr Wirtschaftlichkeit sowie dem Fortwirken überkommener Strukturen. ${ }^{16}$

Offenbar befindet sich das deutsche Stadtheatersystem trotz aller unternommener Anstrengungen auch drei Jahrzehnte nach der schweren, durch die prekäre Lage der öffentlichen Haushalte und die Rückgänge bei den Besuchszahlen bedingten Theaterkrise der unmittelbaren Nachwendezeit in einem fortgesetzten tiefgreifenden Umbruch. Die Zahl der offenen Fragen, Herausforderungen und „institutionellen Aporien“ (Balme 2019, S. 52) ist eher noch größer statt kleiner geworden.

Das zentrale Problem vieler Stadt- und Staatstheater besteht folglich weniger darin, dass sie nicht in der Lage wären, sich mittels diversifizierter Programme, neuer Formen und Formate den neuen Realitäten der vielfältigen Stadtgesellschaft des 21. Jahrhunderts zu stellen. Es besteht vielmehr in dem Fehlen kohärenter Strategien und Steuerungsmodelle, in denen die wichtigen Veränderungen im Bereich der Programme Teil eines sinnvollen Ganzen wären. ${ }^{17}$

Dieses grundlegende Defizit hat durchaus das Potenzial, die angesichts der derzeitigen Situation unsicherer denn je erscheinende Zukunft der deutschen (Stadt-)Theaterlandschaft erheblich zu gefährden.

\section{Literatur}

Althoff, L., E. Priller, und A. Zimmer. 2020. Arbeiten am Stadttheater: Passion als Beruf? Ergebnisse einer empirischen Untersuchung an sechs Theatern in NRW und Ostdeutschland (In diesem Band).

Balme, Christopher. 2019. Die Krise der Nachfolge: Zur Institutionalisierung charismatischer Herrschaft im deutschen Stadt- und Staatstheater. Zeitschrift für Kulturmanagement:

\footnotetext{
${ }^{16}$ Als eines von vielen Beispielen kann neben den baulichen Strukturen die weitgehend ungebrochen praktizierte Herrschaftsform der Intendanz gelten, die, wie Christopher Balme in seinem Aufsatz zur Institutionalisierung charismatischer Herrschaft im deutschen Stadtund Staatstheater nachweist, auf eine in das feudalaristokratische Deutschland des 19. Jahrhunderts zurückreichende, zweifelhafte Tradition zurückgeht, die im 21. Jahrhundert anachronistisch wirke (Balme 2019, S. 42). Dass auch im Jahr 2020 von 142 Häusern 125 von Intendanten und nur 17 von Intendantinnen geleitet werden, wundert vor diesem Hintergrund wenig.

${ }^{17}$ Siehe hierzu Schmidt 2017, Schneider 2013, Fülle 2016. Thomas Schmidt plädiert in diesem Zusammenhang für ein ,systemisches Theatermanagement“. Er versteht darunter „die Leitung und Organisation eines Theaterbetriebes unter Berücksichtigung der Rahmenbedingungen, des gesellschaftlichen Wertewandels und des Theaters als Institution mit einer spezifischen Organisationskultur in einem System anderer Theater und freier Gruppen und Ensembles“ (Schmidt 2017, S. 275).
} 
Kunst, Politik, Wirtschaft und Gesellschaft 2, Hrsg. H. Steffen, T. Martin und C. DeVereaux, 37-54. Bielefeld: Transcript.

Berg, S. 2020. Was sagt uns das, das sogenannte Politische? https://www.nachtkritik.de/ index.php?option=com_content\&view=article\&id=14789:vortrag-sibylle-berg\&catid= 101:debatte\&Itemid=84\#comment-91089. Zugegriffen: 20. Mai 2020.

Brandenburg, Detlef. 2016a. Was also ist des Pudels Kern? Überlegungen zum Werkbegriff. In Wer spielte was? Werkstatistik 2014/15, Hrsg. Deutscher Bühnenverein, 6-9. Hamburg: Inspiring Network.

Brandenburg, Detlef. 2016b. Ins Offen. Die Deutsche Bühne 12:48-51.

Burghardt, Charlotte. 2020. Stadt- und Staatstheater in Bewegung? Zur Rolle von öffentlich subventionierten Theatern in der Stadtgesellschaft und den veränderten Erwartungen des Publikums (In diesem Band).

Deutscher Bühnenverein DBV. 1969. Theaterstatistik 1967/68, Bd. 3. Köln: Deutscher Bühnenverein.

Deutscher Bühnenverein DBV. 1993a. Theaterstatistik 1991/92, Bd. 27. Köln: Deutscher Bühnenverein.

Deutscher Bühnenverein DBV. 1993b. Werkstatistik: Wer spielte was? Deutschland, Österreich, Schweiz 1991/92, 2. Jahrgang. Darmstadt: Mykenae Verlag Rossberg KG.

Deutscher Bühnenverein DBV. 2013. Werkstatistik: Wer spielte was? Deutschland, Österreich, Schweiz 2011/12, 22. Jahrgang. Darmstadt: Mykenae Verlag Erdmann KG.

Deutscher Bühnenverein DBV.2016. Werkstatistik: Wer spielte was? Deutschland, Österreich, Schweiz, 2014/15, 25. Jahrgang. Hamburg: Inspiring Network GmbH \& Co. KG.

Deutscher Bühnenverein DBV. 2019a. Theaterstatistik 2017/18, Bd. 53. Köln: Deutscher Bühnenverein.

Deutscher Bühnenverein DBV. 2019b. Werkstatistik: Wer spielte was? Deutschland, Österreich, Schweiz 2017/18, 28. Jahrgang. Hamburg: Inspiring Network GmbH \& Co. KG.

Deutscher Bühnenverein DBV. 2019c. Verdi und Shakespeare vorn. https://www.buehnenve rein.de/de/28.html?det=535. Zugegriffen: 09. Febr. 2020.

Fülle, Henning. 2016. Freies Theater. Die Modernisierung der deutschen Theaterlandschaft (1960 - 2019). Berlin: Theater der Zeit.

Goebbels, Heiner. 2008. Der Kompromiss ist ein schlechter Regisseur. Theater der Zeit 6:1821.

Haselbach, D., A. Klein, P. Knüsel, und S. Opitz. 2012. Der Kulturinfarkt, von allem zu viel und überall das gleiche. Eine Polemik über Kulturpolitik, Kulturstaat und Kultursubvention. München: Knaus.

Heeg, Günther. 2013. Die AufLösung des Stadttheaters. Die Zukunft des Stadttheaters liegt in einer transkulturellen Theaterlandschaft, In Theater entwickeln und planen. Kulturpolitische Konzeptionen zur Reform der darstellenden Künste, Hrsg. Wolfgang Schneider, 229-243. Bielefeld: Transcript.

Höflich, Joachim R. 2018. Der Opernbesuch als soziale Angelegenheit. In Zur kommunikativen Konstruktion eins, unmöglichen Kunstwerks', In Oper, Publikum und Gesellschaftt, Hrsg. Karl-Heinz. Reuband, 259-284. Wiesbaden: Springer VS.

Höhne, Steffen. 2019. Das System der Darstellenden Künste im Prozess der Transformation. Eine Untersuchung zum Publikum in Deutschland in historischer Perspektive. In Zeitschrift für Kulturmanagement: Kunst, Politik, Wirtschaft und Gesellschaft 5(1): 13-37. 
Israel, Annett. 2009. Entgrenzung. Das aktuelle Erscheinungsbild des Kinder- und Jugendtheaters und seine historischen Bezüge. In Kindertheater Jugendtheater. Perspektiven einer Theatersparte, Hrsg. A. Gronemeyer, J. D. Heße, und G. Taube, 22-45 Berlin: Alexander Verlag.

Michaels, Bianca. 2020. Spielplangestaltung im Kampf um Anerkennung - Diversifizierung als Legitimationsstrategie (In diesem Band).

Reuband, Karl-Heinz. 2011. Das Opernpublikum zwischen Überalterung und sozialer Exklusivität Paradoxe Effekte sozialer Merkmale auf die Häufigkeit des Opernbesuchs. In Jahrbuch für Kulturpolitik 2010/11, Hrsg. Institut für Kulturpolitik der Kulturpolitischen Gesellschaft, 397-406. Essen: Klartext.

Rössel, J., und M. Hoelscher. 2018. Wer geht warum in die Oper? In Sozialstruktur und Motive des Opernbesuchs, In Oper, Publikum und Gesellschaft, Hrsg. Karl-Heinz. Reuband, 241258. Wiesbaden: Springer VS.

Schmidt, Thomas. 2017. Theater, Krise und Reform. Eine Kritik des deutschen Theatersystems. Wiesbaden: Springer VS.

Schneider, Wolfgang. 2013. Under Construction. Reformbedarf auf der Baustelle Theater. In Theater entwickeln und planen. Kulturpolitische Konzeptionen zur Reform der darstellenden Künste, Hrsg. Wolfgang Schneider, 21-26. Bielefeld: Transcript.

Von Cossel, Friederike. 2011. Die Entscheidungsfindung im Kulturbetrieb am Beispiel der Spielplangestaltung im Theater. München: Hampp.

Von Hartz, Matthias. 2011. Kunst oder Kerngeschäft? In Heart of the city. Recherchen zum Stadttheater der Zukunft. Arbeitsbuch 11, Hrsg. Mackert, Josef, Goebbels, Heiner und Barbara Mundel, 30-42, Theater der Zeit 7/8:30-42.

Open Access Dieses Kapitel wird unter der Creative Commons Namensnennung 4.0 International Lizenz (http://creativecommons.org/licenses/by/4.0/deed.de) veröffentlicht, welche die Nutzung, Vervielfältigung, Bearbeitung, Verbreitung und Wiedergabe in jeglichem Medium und Format erlaubt, sofern Sie den/die ursprünglichen Autor(en) und die Quelle ordnungsgemäß nennen, einen Link zur Creative Commons Lizenz beifügen und angeben, ob Änderungen vorgenommen wurden.

Die in diesem Kapitel enthaltenen Bilder und sonstiges Drittmaterial unterliegen ebenfalls der genannten Creative Commons Lizenz, sofern sich aus der Abbildungslegende nichts anderes ergibt. Sofern das betreffende Material nicht unter der genannten Creative Commons Lizenz steht und die betreffende Handlung nicht nach gesetzlichen Vorschriften erlaubt ist, ist für die oben aufgeführten Weiterverwendungen des Materials die Einwilligung des jeweiligen Rechteinhabers einzuholen.

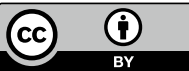

